\title{
Trend and rhythm analysis of time-series data using complex demodulation
}

\author{
HELEN C. SING, DAVID R. THORNE, and FREDERICK W. HEGGE \\ Walter Reed Army Institute of Research, Washington, DC \\ and \\ HARVEY BABKOFF \\ Bar-Ilan University, Ramat Gan, Israel
}

\begin{abstract}
Biological time-series data collected over long intervals generally show combined systematic and periodic fluctuations. Comprehensive analysis of such data requires separation of the trend and rhythmic components. Most available time-series analy tic techniques do not explicitly extract the trend, and do implicitly assume the underlying rhythms are simple symmetrical sinusoids, whose amplitude and phase values remain constant throughout the recorded interval. Neither assumption is very accurate when dealing with biological data, and the stationarity assumption in particular becomes harder to defend as experiments extend over days or even weeks. Complex demodulation (CD) is described here as a technique for separation of trend from cyclic components, and multiple complex demodulation (MCD) as a technique for extraction of all possible frequencies in the data set, along with their moment-by-moment amplitude and phase values.
\end{abstract}

Studies involving sustained performance, cumulative sleep deprivation, temporal isolation, jet lag, work-shift rotations, and the time courses of long-acting drugs are examples of cases in which the presence of biological rhythms can obscure changes in baselines and conceal overall trends. They are also examples of cases in which the biological rhythms themselves are likely to be changing, further complicating analysis and interpretation. In such cases; it would be most desirable to have time-series analytical techniques that can detect the presence of rhythmic components; discriminate them from one another; quantify their individual parameters, preferably at each point in the time series; isolate or separate them from variations in baseline; and identify the proportion of variance accounted for by the trend and each rhythmic component.

Because many of the physiological and psychological experiments in our laboratory are directed toward investigating the dynamic temporal properties of inherent biological rhythms, our choice of time-series analytical techniques has been complex demodulation (CD) and multiple complex demodulation (MCD). These techniques can meet all of the desiderata mentioned above, provided that

\footnotetext{
The opinions or assertions contained herein are the private views of the authors and are not to be construed as official or as reflecting the views of the Department of the Army or the Department of Defense (para 4-3, AR 360-5). Acknowledgment is hereby made to Mr. Joseph Fritz for his continued support in the skillful rendition of the graphic illustrations. Mailing address for H. C. Sing, D. R. Thorne, and F. W. Hegge is: Division of Neuropsychiatry, Walter Reed Army Institute of Research, Washington, DC 20307-5100. Mailing address for H. Babkoff is: Department of Psychology, Bar-Ilan University, 52-100 Ramat Gan, Israel.
}

the time series is longer than the lowest period of interest and is sampled at equal intervals. The basic methodology and applications have been described previously by Orr and Hoffman (1974); Orr, Hoffman, and Hegge (1974, 1976); Redmond, Sing, and Hegge (1982); Sing, Hegge, and Redmond (1984); and Sing, Redmond, and Hegge (1980). Refinement of the procedure is an ongoing process.

Some of the advantages and limitations of CD and alternative analytical techniques have been previously noted in Redmond et al. (1982); Sing, Genser, Babkoff, Thorne, and Hegge (1984); and Sing et al. (1980). Among the advantages of $C D$ and MCD are: (1) the number of data points need not be a power of two; (2) no assumptions about the linearity, monotonicity, or form of the underlying trend are forced; (3) there is no requirement for rhythmic components to be stationary or precisely sinusoidal; and (4) for each point in the time series, amplitude and phase values are provided, from which the timedomain wave form (remodulate) can be constructed. In theory, the choice of an analytical technique is made solely on the basis of the data model and the adequacy with which data meet the assumptions of the model. In practice, however, the choice is often determined by the ease of its application, familiarity to the user, availability on an accessible computer, personal preference, and the desired information to be extracted.

This paper presents an overview of the method and describes the most recent extension of $C D$ and MCD in providing an estimate of the underlying trend. The partitioning of the data series into its constituent trend and cyclic components and calculation of variance accounted for by each also are given. 


\section{METHOD}

A time series may be described as consisting of three components:

$$
x(t)=c(t)+d(t)+e(t)
$$

where $c(t)$ is the trend component, $d(t)$ is the rhythmic component, and $e(t)$ is the error or random component.

Since trend is an "adduced" construct (Sterling \& Pollack, 1968), meaning that it cannot be derived by connecting points on the data set to obtain a smoothed function, it has frequently been estimated by linear or polynomial regression of the entire data set to obtain a best-fitting line in accordance with a least squares criterion. This method offers the quickest and easiest approach but is often difficult to defend. If visual inspection of a graphical display of the data is used, it may not even reveal the general form of the trend, particularly if noise and rhythmic components are large or numerous.

Most techniques for trend extraction fall into one of three categories: (1) regression techniques using an assumed model; (2) time-domain smoothing techniques using single- or multi-pass weighted or unweighted moving averages, medians, and so forth; or (3) frequency-domain smoothing techniques using one or more low-pass filtering operations. Trend extraction by $\mathrm{CD}$ falls into the latter category.

Isolation of trend, $\mathrm{c}(\mathrm{t})$, from rhythm, $\mathrm{d}(\mathrm{t})$ and error, $e(t)$, is achieved with a single-pass $C D$ on the raw data. Subtraction of the trend from the raw data provides the residual data set on which MCD is next applied to obtain the parameters of the various frequencies of interest. A short summary of the basis and of the actual functions used in our $C D$ and MCD techniques is given here. Detailed discussions and illustrations are given in the literature previously cited.

From a descriptive point of view, the MCD technique is analogous to a least squares fit procedure for interpolating between data points, except that a sum of trigonometric functions is the model to be "the best fit." The Euler function provides the rationale for using the trigonometric sine and cosine functions to transform each datum or epoch value from the real to the complex domain. For the moment, we will postpone comparison with Fourier analysis, other than acknowledging that this is the theoretical basis by which transformation between time and frequency domains occurs. The texts of Bloomfield (1976) and Bracewell (1978) provide excellent theoretical foundations, are not mired in inscrutable mathematical notations, and present Fourier analysis in a comprehensible way. Bloomfield also includes FORTRAN computer programs for some of the methods of analysis.

A review of the concept of complex numbers is given here to aid in understanding the use of the Euler function in $C D$. Real numbers are a subset of numbers in the complex-number system and so may also be written in this system's notation. A complex number $(\mathrm{z})$ is composed of a real ( $R e$ ) part (x) and an imaginary (Im) part (jy).
The complex number, $z=x+j y$, may be mapped in the complex plane geometrically (Cadzow, 1973), as shown in Figure 1, where $j=(-1)^{1 / 2}$. A complex number is seen in this representation as a vector from the origin to the point $(x, y)$ with magnitude $|z|$ and direction given by the angle $\theta$ from the $\operatorname{Re}$ axis. The magnitude $|z|$ and the phase angle $\theta$ also may be expressed in more familiar terms as:

$$
\begin{aligned}
|z| & =\left(x^{2}+y^{2}\right)^{1 / 2} \\
\theta & =\operatorname{Arctan} y / x
\end{aligned}
$$

Trigonometric relationships can be used to obtain the following equalities:

$$
\mathrm{x}=|\mathrm{z}| \cos \theta, \text { and } \mathrm{y}=|\mathrm{z}| \sin \theta
$$

The real number $\mathrm{z}$ may then be denoted as:

$$
z=|z|(\cos \theta+j \sin \theta)
$$

The expression in parenthesis is commonly known as the Euler identity.

In the CD method, the original data set, $a_{1}, a_{2}, \ldots, a_{n}$, is transformed to the complex domain by multiplying each datum by sine and cosine functions having the frequency to be examined. Two data series result as follows:

$$
z_{i}(R e)=a_{i} \cos 2 \pi f_{m} t / s
$$

and

$$
\mathrm{z}_{\mathrm{i}}(\mathrm{Im})=\mathrm{a}_{\mathrm{i}} \sin 2 \pi \mathrm{f}_{\mathrm{m}} \mathrm{t} / \mathrm{s}
$$

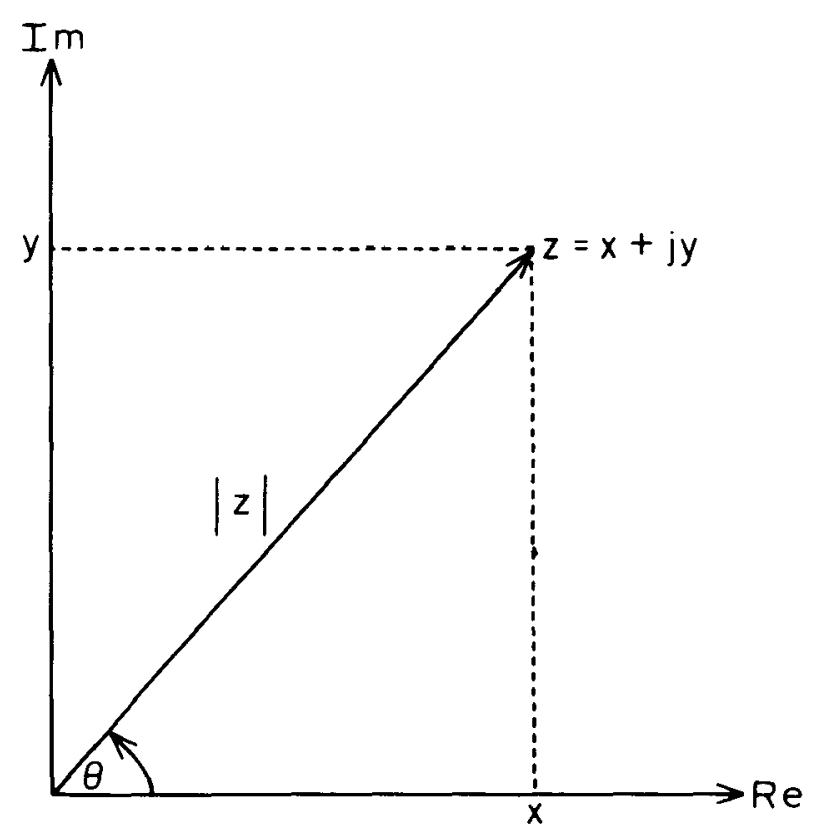

Figure 1. Geometrical mapping of a complex number, $z=x+$ jy, onto the complex plane, where $R e$ is the real axis and Im the imaginary axis and $j=(-1)^{1 / 2}$. 
where $\mathrm{f}_{\mathrm{m}}=$ the $\mathrm{mth}$ frequency selected for demodulation; $\mathrm{i}=1,2 \ldots \mathrm{n} ; \mathrm{t}=\mathrm{i}-1 ; \mathrm{n}=$ number of epochs in the data set; $s=$ number of epochs in the desired period. For circadian rhythmicity, the base period desired would be $24 \mathrm{~h}$, so data sampled at the rate of four times per hour would produce $\mathrm{s}=96$ epochs per $24-\mathrm{h}$ period, and $f_{m}=1$ cycle per period. For this same base period, $f_{m}=2$ would mean a frequency of two cycles per $24-\mathrm{h}$ period.

Implicit in each data value, $a_{i}$, is the complex representation of all frequencies present in the entire time series. However, multiplication by sine and cosine functions generates products that are sums and differences of sines and cosines, resulting in both wanted and unwanted frequencies. The difference frequency wanted is that between the selected modulating frequency and the information in the data series at that same frequency. That difference approaches zero and, indeed, would be zero if the data frequency and demodulating frequency were identical. This information can easily be extracted with an exponential low-pass filter which passes information near zero frequency and rejects the unwanted frequencies.

Figure 2 is a graphic summary of the CD analysis. Figure $2 \mathrm{a}$ shows a time series of heart rate (in beats per 30 min over $72 \mathrm{~h}$ ), which are the input data to $C D$. The data are transformed into real (cosine) and imaginary (sine) values on which are superimposed their filtered outputs (Figures $2 \mathrm{~b}$ and $2 \mathrm{c}$, respectively). Local parameters of power, amplitude, phase, and remodulate for each epoch are obtained from the filtered outputs, $z_{i}^{\prime \prime} s$, as follows:

Power:

$$
P_{i}=2.0\left[z_{i}^{\prime \prime 2}(R e)+z_{i}^{\prime 2}(\operatorname{Im})\right]
$$

Amplitude:

$$
A_{i}=\left(P_{i}\right)^{1 / 2} \text {. }
$$

Phase:

$$
\phi_{\mathrm{i}}=\arctan \left[\mathrm{z}_{\mathrm{i}}^{\prime \prime}(\mathrm{Im}) / \mathrm{z}_{\mathrm{i}}^{\prime \prime}(\mathrm{Re})\right] \text {. }
$$

$$
\text { Remodulate: } \quad \begin{aligned}
\quad R_{i}= & 2.0\left[\mathrm{z}_{\mathrm{i}}^{\prime \prime}(\mathrm{Re}) \cos 2 \pi \mathrm{f}_{\mathrm{m}} \mathrm{t} / \mathrm{s}\right. \\
& \left.+\mathrm{z}_{\mathrm{i}}^{\prime \prime}(\operatorname{Im}) \sin 2 \pi \mathrm{f}_{\mathrm{m}} \mathrm{t} / \mathrm{s}\right] .
\end{aligned}
$$

Figures $2 \mathrm{~d}, 2 \mathrm{e}$, and $2 \mathrm{f}$ show representations of the amplitude, phase, and remodulate, respectively, of the circadian component. Note that the trend has not been removed from these data and that it can be detected in Figures 2a, 2d, and $2 \mathrm{f}$.

For the last five of the six intervals shown in Figure 2e, phase values actually hover around zero along the time axis. Due to a plotting convention, when the curve crosses the axis, it appears to jump abruptly by an amount equivalent to $360^{\circ}$ (points marked " $\wedge$ "). The point marked "**" is assumed to be a true phase shift of undetermined cause.

The remodulate is the smoothed function of the rhythmic component extracted from the data set and retransformed back to the time domain. For a given period, the maximum number of cyclic components determinable (known as the Nyquist frequency limit) is numerically equal to one half the sampling rate. Given a sampling rate of four times per hour, it is possible, theoretically, to extract frequencies from 1 through 48 cycles per $24-h$ period. Whereas $C D$ demodulates only one frequency in the period of interest, MCD steps through and extracts each allowable integral frequency sequentially. All parameters for each epoch are output and stored on disk files along with statistical estimates of variance accounted for and covariances among constituent frequencies.

\section{TREND}

In time series where there is no discernible trend, $\mathrm{MCD}$ may be applied directly to the data set. If a trend is present, it is prudent to remove it before proceeding to rhythmic analysis. Rather than forcing linearity on the data to derive trend, we apply $C D$ to the data in a first pass, setting $\mathrm{s}=\mathrm{n}$ (the total number of data samples or epochs) and $f_{m}=1$. The resulting remodulate in this case is a representation of the inherent trend in the data. It may best be con-
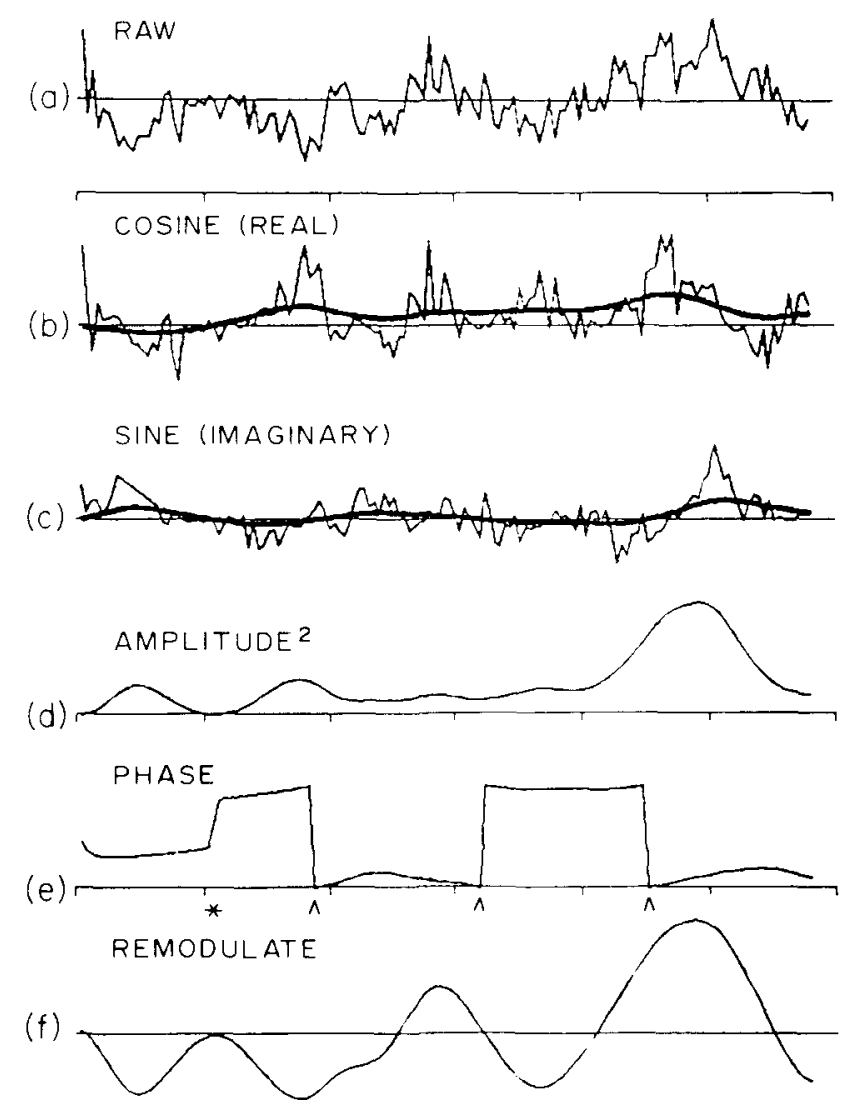

Figure 2. Graphic summary of the CD technique. (a) Sample input data of the original time series consisting of 144,30 -min samples of heart rate over 3 days. (b) Intermediate transformed series generated by multiplication with 24-h cosine function, with filtered output values superimposed. (c) Same as (b) except multiplied with 24-h sine function. (d) Instantaneous power values for the 24-h rhythm. (e) Instantaneous phase values for the 24-h rhythm. (f) Reconstructed waveform of the circadian (24-h) rhythm, which may be compared to the original in (a). 
ceptualized as the zero and near-zero frequency components, though it has a finite bandwidth from zero through the lowest frequency extractable with the given data set and sampling rate.

For example, suppose 3 days of data were collected at 1-h intervals, ultimately to be analyzed by MCD for ultradian, circadian, and infradian rhythms with periods from 2 to $36 \mathrm{~h}$. The first-pass remodulated trend would then consist of the zero-frequency component, that is, a " $D C$ " component equal to the overall mean, plus all very low ferquency components up through a frequency of one cycle per $72 \mathrm{~h}$, if such were actually present. Although the presence of a significant 72 -h rhythm is unlikely with most commonly encountered biological data, this possibility must be kept in mind. Thus, the remodulate extracted from a 28-day sample might include not only the nominal overall "trend," but probably a detectable lunar or estral modulation as well.

A disadvantage of this method in some circumstances is that the characteristics of the smoothing operation (filter bandwidth) are partly determined by the length of the data series being treated. If comparisons are to be made between different equal-length series, or only with the original series itself, this is not a problem. If comparisons are to be made between series whose lengths are not equal or are not small integral multiples, then other smoothing techniques may be preferred.

A second disadvantage in some cases is that the series must be at least twice as long as the lowest period of interest, in order to separate that thythmic component from the trend. Thus, if the longest period of interest is circadian, data must be collected for a minimum of 2 days, This difficulty is more apparent than real. Attempting to assess trend in a single day leads to an inability to discriminate between a change in baseline (i.e., trend) and circadian rhythm amplitude.

A distinct advantage of the method, however, is that the extraction of trend maintains the same scale and metrics as does the extraction of the rhythmic components, aiding comparisons and variance calculations. Another advantage is that both extraction operations are performed with the same program, simply by changing the input parameters.

Once the trend is isolated, it may be fitted to empirical or theoretical equations by any of the available techniques or submitted to further analysis directed at identifying experimental effects or underlying mechanisms.

Figure 3a shows an example of the trend derived from the initial $C D$ pass superimposed on the original raw data.

\section{RHYTHMIC COMPONENTS}

Subtraction of the remodulate values of the trend from the original data leaves rhythmic and random or noise components as the residual. The residual is submitted to MCD in which the period parameter for the sine, cosine argument is now the desired one (e.g., $24 \mathrm{~h}$ ). The circa-

(b)

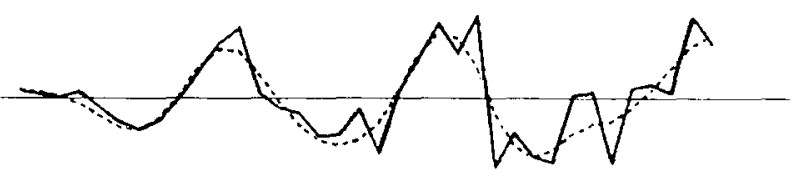

- RESIDUAL

-.-- CIRCADIAN

Figure 3. (a) Original data series with trend superimposed. (b) Residual obtained after subtraction of trend, with circadian component superimposed.

dian component [ 1 cycle per day (cpd)] is extracted for biological data. Other cyclic components, up to one half the sampling rate per $24 \mathrm{~h}$, may be obtained.

In addition to the local parameters for the trend and each cyclic component demodulated, the relative contribution to variance of each is calculated. The first-pass CD determines the variance accounted for by the trend, that is, the percentage contribution of trend relative to the whole data set. The second-pass MCD provides variance of the residual, partitioned according to each rhythmic component.

In practice, the total variance accounted for in the residual by all cyclic components usually approaches or equals $100 \%$. Error or noise, then, is included in one or more of the extracted components and must be identified by other means. The question of whether a derived rhythmic component is real, is due to random noise, or is a combination of both is seldom answered with certainty. The question is most frequently answered by inspection of the phase value for each epoch. Random cyclic components in general have randomly varying phase values. If phase values show stability, linear drift, or slow variations, then the assumption is that this is a true component of the data. Other decision guidelines involve inspection of absolute amplitude, amplitude relative to neighboring components, and preknowledge of experimenal manipulations or underlying mechanisms. 


\section{DISCUSSION}

Box and Jenkins (1976) have differentiated between deterministic and stochastic time series, defining the former as being symmetrical with single frequency, amplitude, and phase predictable over time, and defining the latter as having future values describable only in terms of a probability distribution. By this definition, complex demodulation is applicable to semistationary time series with slowly changing phase or amplitude or both, that is, stochastic series with known or empirically measurable distributions. Additionally, by using the remodulate, dynamic changes in each component may be observed, provided the time series encompasses more than one complete period. The use of symmetric functions applicable to deterministic series has not provided acceptably good fits to our data. The implicit assumption of stationarity has not been supported either, as may be seen in the accompanying figures.

A capsule comparison of several time-series techniques may be helpful at this point. (1) Most of the commonly used regression-based techniques that fit a sine wave to the data provide a single average amplitude value and a single average phase value for one selected or "best-fit" frequency. (2) Fourier analysis provides a single average amplitude value and a single average phase value for
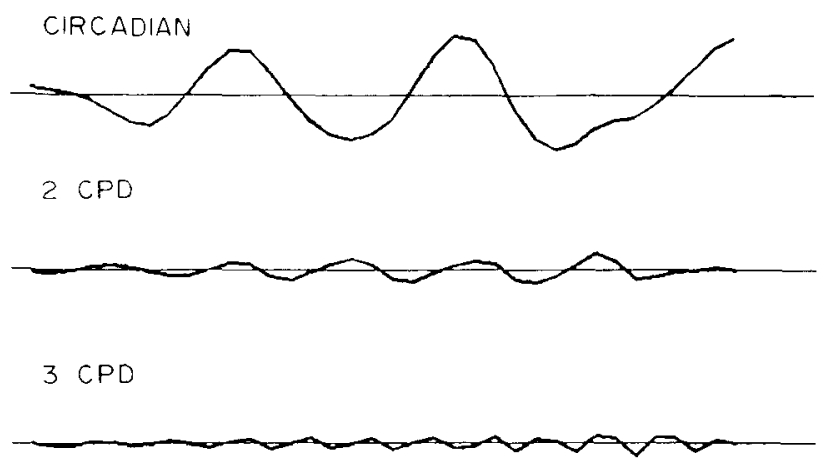

$4 \mathrm{CPD}$

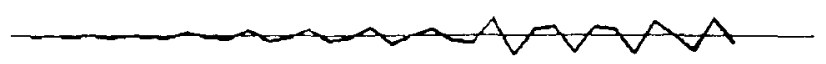

5 CPD

6 CPD

Figure 4. Circadian, 2 cycles per day (cpd), 3 cpd, 4 cpd, 5 cpd, and $6 \mathrm{cpd}$ rhythmic components of residual. Ordinate is relative amplitude.

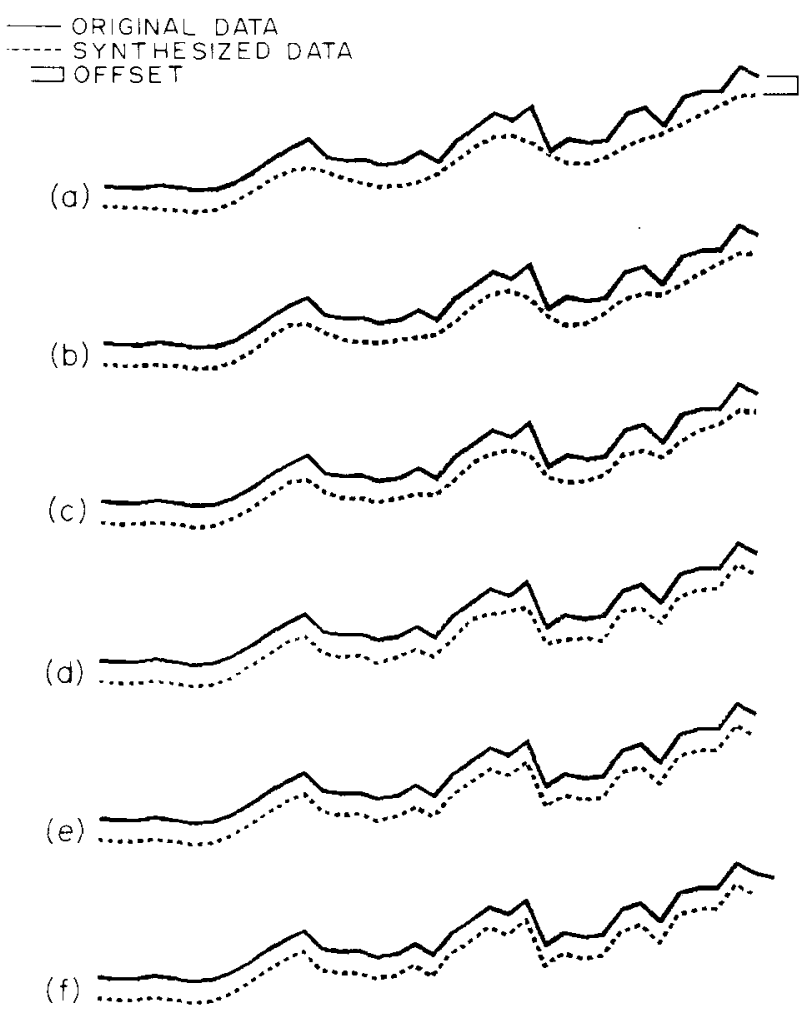

Figure 5. Trend plus sequential addition of the rhythmic components from Figure 4 showing successive approximation and recovery of the original waveform. (a) Summation of circadian with trend. (b) Summation of (a) with 2 cpd. (c) Summation of (b) with 3 cpd. (d) Summation of (c) with 4 cpd. (e) Summation of (d) with 5 cpd. (f) Summation of (e) with 6 cpd. Sum of trend and all rhythmic components demodulated.

each of $n / 2$ different frequencies (where $n$ is a power of two close to or equal to the number of data epochs). (3) Single CD provides $n$ instantaneous amplitude values and $n$ instantaneous phase values, hence $n$ time domain remodulate values for one selected frequency. (4) Multiple $C D$ provides these same $n$ values for each of $m$ harmonically related frequencies (where $m$ is a number determined by the sampling rate, record length, and major period of interest, with an upper limit of $n / 2$ ).

$C D$ and MCD are of potential use in the following situations: (1) When the major period or frequency of interest is known in advance or is determined by preanalysis with one of the other techniques. (2) When there is a particular interest or need to know how the amplitude or phase of the rhythm interrelates to other components, varies over time, or responds to an experimental manipulation or identified event. (3) When the rhythm is viewed as an identifiable source of "noise" obscuring other effects of interest, and its removal is desired. (4) Where one or more of the above is the case, and it is also desired to identify and/or remove the overall trend.

The major difference between MCD and Fourier analysis, is in the output results. Whereas Fourier analysis 
provides only global parameters (i.e., single-value power and phase, over the entire time series, even if the number of data points extends over more than one complete period), MCD provides a power, phase, and remodulate value for each data point. Thus, components may be studied in detail, instabilities in a rhythm may be ascertained and exactly located in time, the emergence and decay of a rhythm may be detected, and direct comparisons can be made among different rhythms in the same or other related data series. In short, MCD provides a wealth of information giving details at each epoch in the time series not obtainable by other methods.

\section{EXAMPLE}

Specific applications of CD and MCD are described in Babkoff, Genser, Sing, Thorne, and Hegge (1985); Babkoff et al. (1985); and Thorne, Genser, Sing, and Hegge (1983). A graphical example of the CD and MCD technique is presented here to illustrate how a time series is decomposed into both sets of constituents and how the original data may be reconstructed by linear summation of the constituents. Thus, a representational model of the data is realized.

The dataset is from a 72-h sleep deprivation study and is a measure of response lapses in a lexical decision task taken at alternate hours (Babkoff, Genser, Sing, Thorne, \& Hegge, 1985). The original data series is plotted in Figure $3 \mathrm{a}$ and shows an unmistakable upward trend. The "spikiness" of the curve is indicative of the presence of rhythmic components. After a first-pass $\mathrm{CD}$, a function representing the trend is isolated from the rest of the data and is shown superimposed on the original data in Figure 3a. The residual after subtraction of the trend from the original data is shown in Figure 3b; the derived circadian component is plotted superimposed on the residual. The second MCD pass decomposes the residual into the constituent rhythmic components shown in Figure 4 as circadian, 2 cpd (once every 12-h cycle), 3 cpd (8-h cycle), $4 \mathrm{cpd}$ (6-h cycle), $5 \mathrm{cpd}$ (4.8-h cycle), $6 \mathrm{cpd}$ (4-h cycle). The latter is the limit of determinable frequencies with the given sampling rate and base period. The remodulates are not pure symmetrical sine (or cosine) waves, but have varying amplitude.

The reconstruction of the original data in stepwise manner is shown in the series of panels in Figure 5. The circadian is summed with the trend to produce the broken

Table 1

Variance Accounted for by Trend and Rhythmic Components in the Entire Data Set

\begin{tabular}{ll}
\hline Component & $\mathbf{R}^{2}$ \\
\hline Trend & 0.8570 \\
Circadian & 0.1069 \\
$2 \mathrm{cpd}$ & 0.0104 \\
$3 \mathrm{cpd}$ & 0.0112 \\
$4 \mathrm{cpd}$ & 0.0105 \\
$5 \mathrm{cpd}$ & 0.0038 \\
$6 \mathrm{cpd}$ & 0.00007 \\
\hline
\end{tabular}

Table 2

Variance Accounted for by Rhythmic Components in Residual

\begin{tabular}{ll}
\hline Component & $\mathbf{R}^{2}$ \\
\hline Circadian & 0.7478 \\
$2 \mathrm{cpd}$ & 0.0726 \\
$3 \mathrm{cpd}$ & 0.0782 \\
$4 \mathrm{cpd}$ & 0.0731 \\
$5 \mathrm{cpd}$ & 0.0269 \\
$6 \mathrm{cpd}$ & 0.0005 \\
\hline
\end{tabular}

curve in Figure 5a, followed by addition of $2 \mathrm{cpd}, 3 \mathrm{cpd}$, $4 \mathrm{cpd}, 5 \mathrm{cpd}$, and $6 \mathrm{cpd}$ in Figures $5 \mathrm{~b}$ through 5f, respectively. The original data are plotted in solid lines displaced five units upward to provide a direct comparison to the stepwise reconstructed model.

Table 1 lists the variance accounted for by the trend and that accounted for by each of the rhythmic components in the original data. The variance accounted for by each cyclic component in the residual is given in Table 2.

Data from sustained operations experiments, which by their very nature extend over multiple base periods, are ideally suited to analysis by $C D$ and MCD. Long-term trends and rhythmic components of diverse measures may be directly compared and/or correlated at any time epoch. Additionally, changes of state and the occurrence of unusual events can be detected and precisely localized.

Details of the computer program used for MCD analysis, details of use, and calibration will be the subject of a future publication.

\section{REFERENCES}

Babkoff, H., Genser, S. G., Sing, H. C., Thorne, D. R., \& Hegge, F. W. (1985). The effects of progressive sleep loss on the lexical decision task: Response lapses and response accuracy. Behavior Research Methods, Instruments, \& Computers, 17, 614-622.

Babkoff, H., Thorne, D. R., Sing, H. C., Genser, S. G., Taube, S. L., HegGE, F. W. (1985). Dynamic changes in work/rest duty cycles in a study of sleep deprivation. Behavior Research Methods, Instruments, \& Computers, 17, 604-613.

Bloomfield, P. (1976). Fourier analysis of time series: An introduction. New York: Academic Press.

Box, G. E. P., Jenkins, G. M. (1976). Time series analysis: Forecasting and control. San Francisco: Holden-Day.

BRACEWELL, R. N. (1978). The Fourier transform and its applications. New York: McGraw-Hill.

CAOzow, J. A. (1973). Discrete time sustems: An introduction with interdisciplinary applications. Englewood Cliffs, NJ: Prentice-Hall.

ORR, W. C., \& HofFMAN, H. J. (1974). A 90-minute cardiac biorhythm: Methodology and data analysis using modified periodograms and complex demodulation. IEEE Transactions Biomedical Engineering, 21, 130-143.

OrR, W. C., Hoffman, H. J., \& Hegge, F, W. (1974). Ultradian rhythms in extended performance. Aerospace Medicine, 45, 995-1000.

OrR, W. C., HoffmaN, H. J., \& Hegge, F. W. (1976). The assessment of time-dependent changes in human performance. Chronobiologia, 8, 293-305.

Redmond, D. P., Sing, H. C., \& Hegge, F. W. (1982). Biological time series analysis using complex demodulation. In F. W. Brown \& R. C. Graeber (Eds.), Rhythmic aspects of behavior. Hillsdale, NJ. Erlbaum.

Sing, H. C., Genser, S. G., Babkoff, H., Thorne, D. R., \& Hegge, F. W. (1984). Complex demodulation-A technique for assessing periodic components in sequentially sampled data. Proceedings of the 29 th conference on design of experiments in Army research, development 
and testing, Uniformed Services University of the Health Sciences, Bethesda, MD. Department of Defense, Army Mathematics Steering Committee for the Chief of Research, Development and Acquisition (ARO Report 84-1).

Sing, H. C., Hegge, F. W., \& Redmond, D. P. (1984). Complex demodulation-Technique and application. In E. Haus \& H. F. Kabat (Eds.), Chronobiology 1982-1983. Basel, Switzerland: Karger.

Sing, H. C., Redmond, D. P., \& Hegge, F. W. (1980). Multiple complex demodulation: A method for rhythmic analysis of physiological and biological data. Proceedings of the 4th Annual symposium on com- puter applications in medical care (pp. 151-158). New York: Institute of Electrical and Electronics Engineers.

Sterling, T. D., \& Pollack, S. V. (1968). Introduction to statistical data processing. Englewood Cliffs, NJ: Prentice-Hall.

Thorne, D. R., Genser, S. G., Sing, H. C., \& Hegge, F. W. (1983). Plumbing human performance limits during 72 hours of high task load. In S. E. Forshaw (Ed.), Proceedings of the 24th Defence Group Seminar on the human as a limiting element in military systems:Volume 1 (NATO-DRG Report No. DS-A-DR [83] 170, pp. 17-41). Toronto, Canada: NATO Defence Research Group. 